\title{
Corrections
}

\section{The BKZ as an alternative to the Wagner model for fitting shear and elongational flow data of an LDPE melt}

\author{
R. G. Larson and K. Monroe
}

AT \& T Bell Laboratories, Murray Hill, N.J. (U.S.A.)

Rheol. Acta, 23, 10-13 (1984)

It was claimed that a separable BKZ model, with a potential function given in Eq. (21), fits the damping function in uniaxial extension and shear for Melt I, as well as the Wagner model fits these damping functions. The plot of the fit for uniaxial extension, figure 2 of Larson and Monroe, however, is in error; the fit is actually poor. A potential function, $u$, that does yield a good fit, not only to shear and uniaxial extensional data, but to biaxial [1] as well, is

$$
u=\frac{3}{2 \xi} \log \left[1+\frac{\xi}{3}(I-3)\right] \text {, }
$$

where

$$
\xi \equiv c_{0}+c_{2} \operatorname{atan}\left[\frac{c_{1}\left(I_{2}-I_{1}\right)^{3}}{1+\left(I_{2}-I_{1}\right)^{2}}\right]
$$

and

$$
I \equiv(1-\alpha)\left(I_{1}-3\right)+\left(1+2 \alpha\left(I_{2}-3\right)\right)^{1 / 2}+2
$$

with $c_{0}=0.20, c_{1}=0.05, c_{2}=0.121$, and $\alpha=0.1 . I_{1}$ and $I_{2}$ are the first and second invariants of the Finger strain tensor. The damping functions calculated from this potential function are compared to the experimental damping functions in figure 1 . The correct amount of strain softening is predicted for all three types of deformation; the Wagner damping function fitted to uniaxial extension predicts too much strain softening in biaxial extension [2].

The Wagner model also predicts a zero second normal-stress difference. Eqs. (1-3) give a negative second normal-stress difference; the predicted ratio of

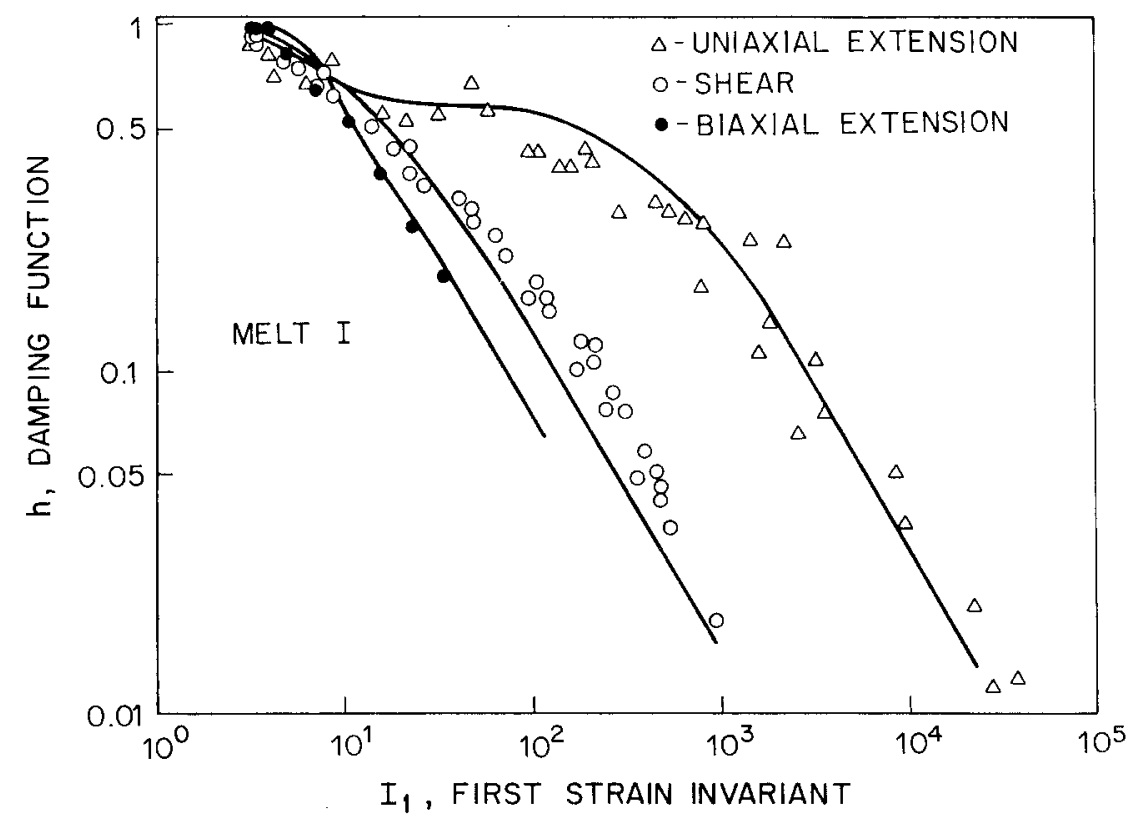

Fig. 1. Measured damping function (symbols) for Melt I compared to the damping function calculated (lines) from Eqs. $(1-3)$ 
second to first normal-stress differences decreases in magnitude from -0.11 at low shear rates to near zero at high shear rates. The magnitude of the second normal-stress difference, $N_{2}$, is roughly proportional to the value of the parameter, $\alpha$. For moderate and large $I_{1}, h_{\text {biax }}<h_{\text {uniax }}$. There is, however, a predicted region at small $I_{1}$ in figure 1 , where $h_{\text {biax }}>h_{\text {uniax }}$. Such a region must occur at small strains if $N_{2}$ is negative. The size of this region grows with $\alpha$ and hence with the magnitude of $N_{2}$. The experimental data in Fig. 1 indicate that the size of this region is small, which is consistent with a small magnitude for $\mathrm{N}_{2}$.

\section{References}

1. Khan SA, Prud'homme RK, Larson RG (1987) Rheol Acta 26:144

2. Soskey PR, Winter HH (1985) J Rheol 29:493

(Received December 23, 1986)

Authors' addresses:

Dr. R. G. Larson*)

AT \& T Bell Laboratories

600 Mountain Avenue

Murray Hill, NJ 07974 (U.S.A.)

Miss K. Monroe

Rohm and Haas, Tn, Inc. Knoxville, TN 37921

(U.S.A.)

*) To whom correspondence should be addressed 